\title{
Editorial: Optical Microscopic and Spectroscopic Techniques Targeting Biological Applications
}

\author{
Vicente Micó ${ }^{1}$, Giancarlo Pedrini ${ }^{2}$, Ming Lei ${ }^{3}$, Chao Zuo ${ }^{4}$ and Peng Gao ${ }^{5 *}$ \\ ${ }^{1}$ Departamento de Óptica y de Optometría y Ciencias de la Visión, Universidad de Valencia, Burjassot, Spain, ${ }^{2} I n s t i t u t$ für \\ Technische Optik, Universität Stuttgart, Stuttgart, Germany, ${ }^{3}$ School of Physic, Xi'an Jiaotong University, Xi'an, China, ${ }^{4}$ School of \\ Electronic and Optical Engineering, Nanjing University of Science and Technology, Nanjing, China, ${ }^{5}$ School of Physics and \\ Optoelectronic Engineering, Xidian University, Xi'an, China
}

Keywords: optical microscopy, spectroscopy, super-resolution, deep-learning, quantitative phase imaging, fluorescent probe, algorithms

\section{Editorial on the Research Topic}

Optical Microscopic and Spectroscopic Techniques Targeting Biological Applications

Optical microscopy and spectroscopy are two mainstream tools for the exploration of an unknown microworld, especially in biomedical fields. Specifically, optical microscopy uses visible light and a system of lenses to magnify images of small samples [1]. It is non-invasive to live samples and has the capability to visualize specific structures once employing fluorescent labeling strategies [2]. Meanwhile, optical spectroscopy allows investigation of chemical, physical and electronic structures of matter at atomic, molecular, and macro scales [3]. In the past decades, remarkable developments of optical microscopy and spectroscopy have been witnessed, mainly in hardware implementation, algorithm performance, and innovative approaches [4-8].

We are glad to see that this special issue collects 23 articles, which report both the latest technological advances and the applications of optical microscopy and spectroscopy. Conceptually, these articles can be categorized into five classes according to the specific techniques (Figure 1).

The first class is fluorescence microscopy and correlation spectroscopy. These techniques can visualize the structures or the bio-molecular dynamics of samples by tagging them with fluorescence markers. Specifically, Li et al. review 3D imaging with dual-lens fluorescence microscopy, Qin and $\mathrm{Xia}$ present simultaneous two-photon fluorescence microscopy with two endogenous fluorescent coenzymes, entitled NADH and FAD. Yu et al. reviews fluorescence correlation spectroscopy (FCS), a powerful technique for quantification of molecular dynamics. Cui et al. fabricated a novel nearinfrared fluorescent nanoparticles (NPs) for high-contrast and high-penetration in-vivo imaging.

The second class is quantitative phase microscopy (QPM). These techniques explore the phase of the light passing through or being reflected by a sample, providing quantitative information of $3 \mathrm{D}$ profiles or refractive index distributions of the sample. Picazo-Bueno et al., Guo et al., and Karako et al. propose new types of digital holographic microscopy (DHM) to quantify the thickness and refractive index distributions of samples. Meanwhile, Lima and Cojoc utilize DHM for the assessment of human neutrophil differentiation from myeloid cells. Zhou et al. propose a noninterference QPM strategy, recovering the phase from a stack of through-focus intensity images. Soto et al. present a partially coherent illumination based optical diffraction tomography (ODT) approach, allowing 3D refractive-index imaging of dynamic samples. Wu et al. propose a lensfree on-chip microscopy incorporated with a high-precision autofocusing algorithm for pixel-superresolved QPM imaging of the sample. Sahu and Mazumder summarize some of the application of adaptive optics (AO) in two-photon fluorescence (TPF) microscopy for wavefront corrections in brain imaging and ophthalmoscopy. 


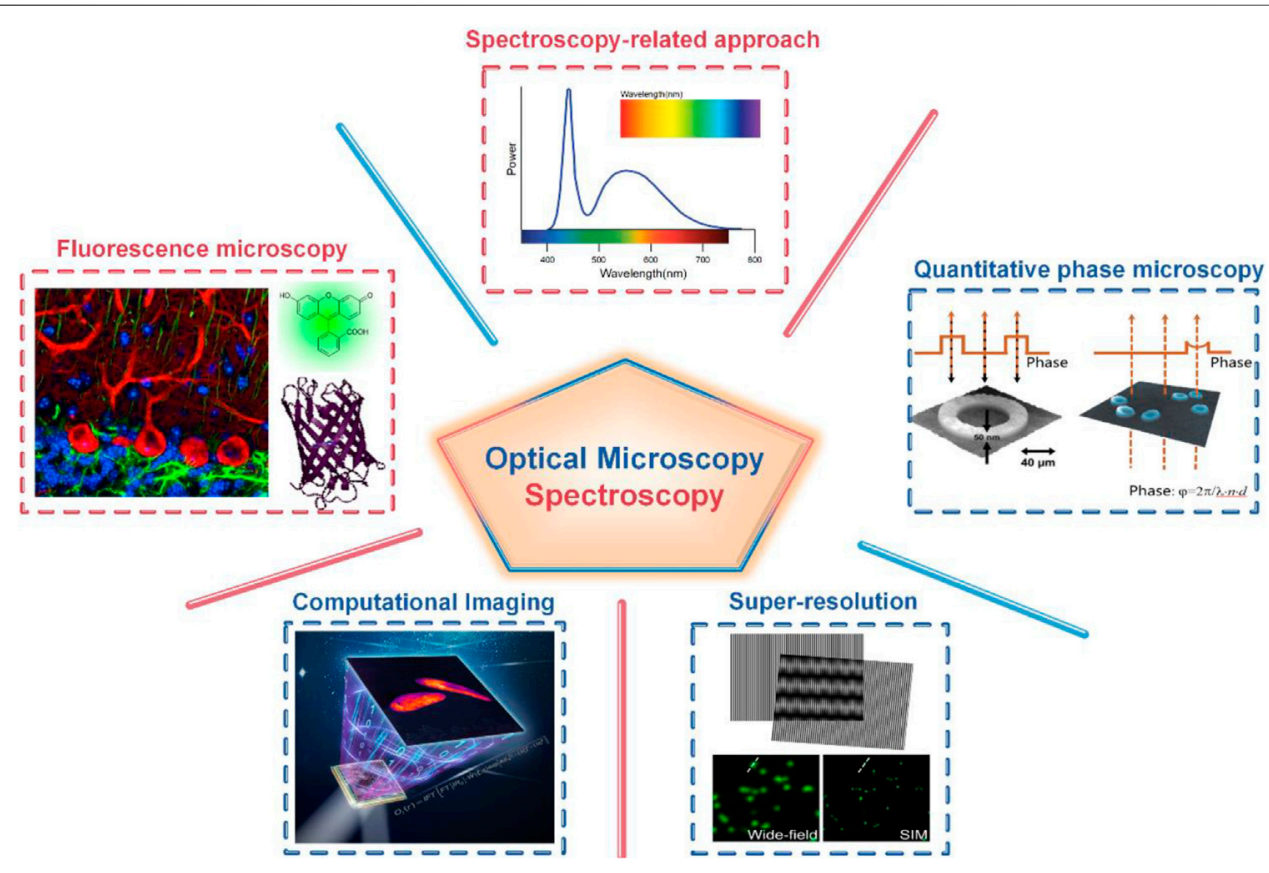

FIGURE 1 | Topical classification of the articles in the special issue [9][10].

The third class is super-resolution optical microscopy. The techniques of this class are far-field, minimally invasive, and they can image samples with a resolution surpassing the diffraction limit. Yong et al. present a high-dynamic-range structured illumination microscopy (HDR-SIM) using a multi-exposure acquisition strategy. With HDR-SIM, microspheres and vesicles with 420 intensity levels can be visualized in the same scene. Wen et al. demonstrate a digital micromirror device (DMD) based optical microscopic apparatus for dual-modality imaging, including quantitative differential phase contrast (qDIC) imaging and coherent SIM imaging. Zhao et al. review recent advances in high-speed SIM that include both hardware and software improvements, including reduction of the number of raw images, GPU acceleration, deep learning, and spatial domain reconstruction. Chen et al. present a super-resolution optical microscopy entitled expansion microscopy (ExM). This technique immobilizes the fluorescent molecules of interest in a polyacrylamide hydrogel. Then, the structure of the sample is spatially amplified as the hydrogel physically expands isotopically, thus indirectly improving resolution. This paper also present the applications of super resolution expansion microscopy in yeast.

The fourth class is deep-learning based computational imaging techniques. These techniques brought about new revolutionary computational power in optical microscopy. Zhang et al. present deep learning (DL) based adaptive optics (AO) to correct optical aberrations. They utilize a revised ResNet-34 network to infer the phase distortions of all the
224 valid zones on a SLM in one shot. Zhang et al. present a new deep-learning-based approach for recovering halo-free white-light diffraction phase images. The neural networkbased method can accurately and rapidly remove the halo artifacts, not relying on any a-priori knowledge. Di et al. present an optimized structural convolution neural network PhaseNet for the reconstruction of digital holograms for DHM.

The fifth class is spectroscopy-related techniques. These techniques can non-invasively probe the structure, properties, and dynamics of molecules in different environments or different physico-chemical conditions. Hence, they are widely used in physics, astronomy, chemistry, biology, and related research fields. Here, $\mathrm{Li}$ et al. review the principle, configuration, and applications of coherent anti-stokes Raman scattering (CARS) microscopy, which can provide high-resolution, high-sensitivity, and non-invasive imaging of specific biomolecules without fluorescent labeling. Poulen et al. review the application of CARS in the identification and characterization of myelin in the mammalian nervous system of different species. Duadi et al. investigate near-infrared (NIR) measurements of turbid media using different size detectors at different positions. GarcíaMartínez et al. propose a supercontinuum (SC) laser source from 450 to $1,600 \mathrm{~nm}$ with programmable spectrum by using liquid-crystal on silicon (LCoS) spatial light modulators (SLM).

To sum up, this research topic features 23 excellent articles encompassing the start-of-art developments and applications of optical microscopy and spectroscopy. This collection should be of interest to readers in the areas of optics, biophysics, and chemistry orientated subjects. It is our 
hope that this special research topic will contribute to the scientific advancements of optical microscopy and spectroscopy, as well as to their practical applications in biology and medicine.

\section{REFERENCES}

1. Girkin J. A Practical Guide to Optical Microscopy. 1st ed. Boca Raton, FL: CRC Press (2019).

2. Dean KM, and Palmer AE. Advances in Fluorescence Labeling Strategies for Dynamic Cellular Imaging. Nat Chem Biol (2014) 10:512-23. doi:10.1038/nchembio.1556

3. Atkin JM, and Raschke MB. Optical Spectroscopy Goes Intramolecular. Nature (2013) 498:44-5. doi:10.1038/498044a

4. Bacia K, Kim SA, and Schwille P. Fluorescence Cross-Correlation Spectroscopy in Living Cells. Nat Methods (2006) 3:83-9. doi:10.1038/nmeth822

5. Cheng J-X, Volkmer A, and Xie XS. Theoretical and Experimental Characterization of Coherent Anti-Stokes Raman Scattering Microscopy. J Opt Soc Am B (2002) 19:1363-75. doi:10.1364/JOSAB.19.001363

6. Lippincott-Schwartz J, and Manley S. Putting Super-Resolution Fluorescence Microscopy to Work. Nat Methods (2009) 6:21-3. doi:10.1038/nmeth.f.233

7. Lichtman JW, and Conchello J-A. Fluorescence Microscopy. Nat Methods (2005) 2:910-9. doi:10.1038/nmeth817

8. Micó V, Zheng J, Garcia J, Zalevsky Z, and Gao P. Resolution Enhancement in Quantitative Phase Microscopy. Adv Opt Photon (2019) 11:135-214. doi:10.1364/AOP.11.000135

\section{AUTHOR CONTRIBUTIONS}

All authors listed have made a substantial, direct, and intellectual contribution to the work, and approved it for publication.

9. Kemper B, and von Bally G. Digital Holographic Microscopy for Live Cell Applications and Technical Inspection. Appl Opt (2008) 47:A52-A61. doi:10.1364/AO.47.000A52

10. Lyncée tec. Available at: https://www.lynceetec.com/types/downloads/

Conflict of Interest: The authors declare that the research was conducted in the absence of any commercial or financial relationships that could be construed as a potential conflict of interest.

Publisher's Note: All claims expressed in this article are solely those of the authors and do not necessarily represent those of their affiliated organizations, or those of the publisher, the editors and the reviewers. Any product that may be evaluated in this article, or claim that may be made by its manufacturer, is not guaranteed or endorsed by the publisher.

Copyright (c) 2021 Micó, Pedrini, Lei, Zuo and Gao. This is an open-access article distributed under the terms of the Creative Commons Attribution License (CC BY). The use, distribution or reproduction in other forums is permitted, provided the original author(s) and the copyright owner(s) are credited and that the original publication in this journal is cited, in accordance with accepted academic practice. No use, distribution or reproduction is permitted which does not comply with these terms. 